\title{
The prospects on the absolute proton beam polarimetry at EIC
}

\author{
A. A. Poblaguev*; A. Zelenski, and G. Atoian \\ Brookhaven National Laboratory, Upton, NY 11973, USA \\ E-mail: poblaguev@bnl.gov
}

In the high-luminosity Electron Ion Collider (eRHIC) proposal the number of ion bunches will be increased and the bunch spacing will be reduced from current $107 \mathrm{~ns}$ (RHIC) to $10.1 \mathrm{~ns}$. This beam timing structure is expected to be a challenge for the elastic events identification in the RHIC CNI (Coulomb Nuclear Interference) polarimeters and, thus, require an upgrade of the polarimeters. It this paper, we discuss possible solutions of the problem for the Polarized Atomic Hydrogen Gas Jet Target designed to measure absolute average proton beam polarization at RHIC.

The 18th International Workshop on Polarized Sources, Targets, and Polarimetry, PSTP2019

23-27 September, 2019

Knoxville, Tennessee

\footnotetext{
*Speaker.

$\dagger$ This manuscript has been authored by employees of Brookhaven Science Associates, LLC under Contract No. DE-SC0012704 with the U.S. Department of Energy.
} 


\section{Introduction}

Precision measurement of the electron and ion beam polarization is important for the future Electron Ion Collider (EIC). Since the Relativistic Heavy Ion Collider (RHIC) rings will be used to accelerate and store the ion beams at EIC [1] and since the proposed hadron beam energies and bunch intensities at EIC are similar to those at RHIC Spin Program [2], the RHIC experience in the proton beam polarization measurements is relevant for the EIC polarimetry design.

At RHIC, the absolute proton beam polarization, which is about $P_{\text {beam }} \sim 55-60 \%$, can be determined [3] with low systematic uncertainties of

$$
\sigma_{P}^{\text {syst }} / P \lesssim 0.5 \%
$$

This result well satisfies the EIC requirements. However, reducing bunch spacing from $107 \mathrm{~ns}$ at RHIC to $10 \mathrm{~ns}$ at EIC may be a challenge.

The goal of this paper is to project our experience in absolute polarization measurements of proton beams at RHIC to EIC. For evaluations, we used the $255 \mathrm{GeV}$ proton beam data acquired in RHIC Run 17 [4].

\section{HJET polarimeter at RHIC}

The Polarized Atomic Hydrogen Gas Target [5] (HJET) is employed to measure the RHIC proton beam absolute polarization since 2004. The scheme of measurements is sketched in Fig. 1. The vertically polarized proton beam, consisting of alternating spin up/down bunches, crosses the vertically polarized hydrogen gas jet and the recoil protons are counted in the silicon detectors. The jet spin is reversed every $5 \mathrm{~min}$. The polarizations of both, so-called blue and yellow, RHIC beams are measured simultaneously and continuously.

The beam $a_{\mathrm{N}}^{\text {beam }}$ and jet $a_{\mathrm{N}}^{\text {jet }}$ single spin asymmetries can be expressed via the elastic event counts $N_{L R}^{\uparrow \downarrow}$ discriminated by the beam or jet spin $(\uparrow \downarrow)$ ) and by the left/right $(L R)$ location of the detectors relative to the beam direction:

$$
a_{\mathrm{N}}=\frac{\sqrt{N_{R}^{\uparrow} N_{L}^{\downarrow}}-\sqrt{N_{L}^{\uparrow} N_{R}^{\downarrow}}}{\sqrt{N_{R}^{\uparrow} N_{L}^{\downarrow}}+\sqrt{N_{L}^{\uparrow} N_{R}^{\downarrow}}}=\left\langle A_{\mathrm{N}}\right\rangle P
$$

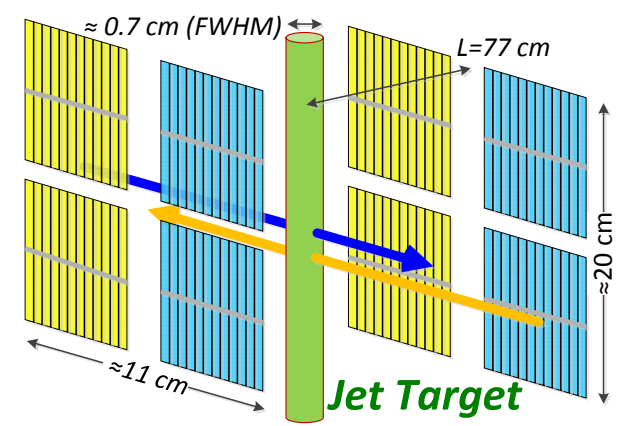

Figure 1: A schematic view of HJET polarimeter. 8 Silicon detectors, 12 readout channels each, are optionally referred as blue and yellow depending on which RHIC beam they measure. The waveform fit, done for every detected event, accurately determines the recoil proton kinetic energy $T_{R}$ and time of flight (TOF). The vertically oriented Si strips tag the recoil angle $\theta_{R}$. 

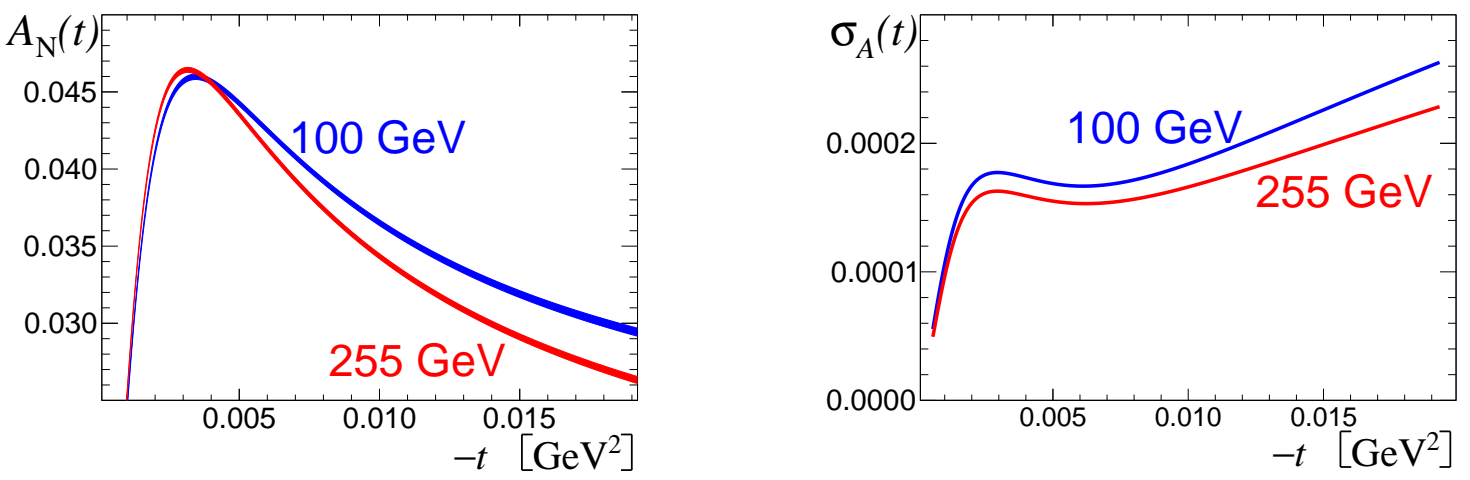

Figure 2: Left: the elastic $p p$ analyzing power [3] measured at HJET. Right: the experimental uncertainties (stat+syst) in these measurements.

where $P$ is the beam or jet polarization and $\left\langle A_{\mathrm{N}}\right\rangle$ is the average analyzing power in the measurement.

For elastic $p p$ scattering, $\left\langle A_{\mathrm{N}}\right\rangle$ is the same for $a_{\mathrm{N}}^{\text {beam }}$ and $a_{\mathrm{N}}^{\text {jet }}$. Therefore, the beam polarization $P_{\text {beam }}$ can be related,

$$
P_{\text {beam }}=\frac{a_{\mathrm{N}}^{\text {beam }}}{a_{\mathrm{N}}^{\text {jet }}} P_{\text {jet }},
$$

to the well determined jet polarization $P_{\text {jet }}=0.957(1)$ [5].

The developed background subtraction method [6] provides reliable isolation of the elastic $p p$ events and, thus, reduces systematic uncertainties in the beam polarization measured to the value given in Eq. (1.1). For 8-hour RHIC store in Run 17, a typical result for the measured average beam polarization was

$$
P_{\text {beam }} \approx\left(55 \pm 2.0_{\text {stat }} \pm 0.3_{\text {syst }}\right) \% .
$$

Asymmetry (2.1), measured as function of the momentum transfer $t=-2 m_{p} T_{R}$, allowed us to precisely determine [3] the elastic $p p$ analyzing power for two proton beam energies, 100 and 255 $\mathrm{GeV}$. The obtained results are displayed in Fig. 2.

\section{HJET data analysis}

For HJET measurements at RHIC, a typical time/amplitude distribution in a Si strip is shown in Fig. 3. The data rate is strongly dominated by the prompts, the fast particle penetrating the Si strips and, thus, identified by low deposited energy and small time of flight (TOF). The recoil protons with energy above $7.8 \mathrm{MeV}$ punch through a Si strip and, thus, only part of the kinetic energy is detected. Such events as well as prompts might be a serious problem for beam polarization measurement at EIC. Due to the frequent, $10 \mathrm{~ns}$, bunch spacing at EIC, the elastic pp signals might be mismatched with punch-trough protons and background events (in particular, prompts) from other bunches.

In the HJET data analysis, it was found that, for the same detected energy, the signal waveform shape for the stopped and punch-through protons is noticeably different [6]. Therefore, analyzing the signal waveform shape, one can recognize the punch-through proton and reconstruct it kinetic energy. 

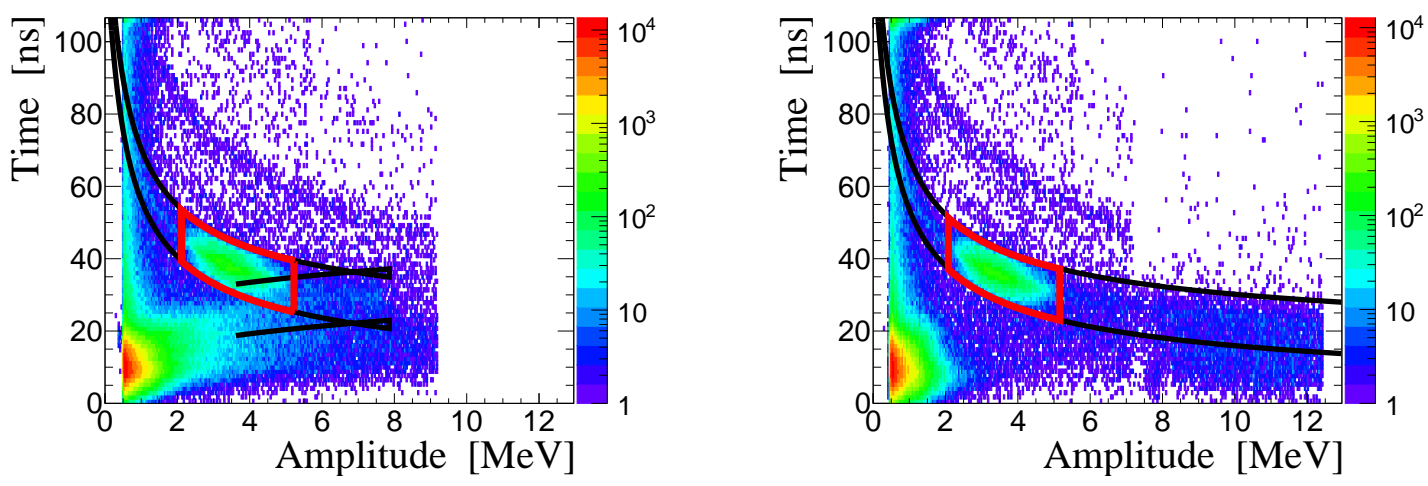

Figure 3: Left: a typical detected signal time/amplitude distribution in a Si strip. Black lines specify the time/amplitude area corresponding to the recoil protons. Red contour isolates the elastic $p p$ events. Right: the same distribution after reconstruction of kinetic energy of the punch-trough protons.

Critically important element of the HJET data analysis is the background subtraction [6]. The method is based on a hypothesis that background have the same energy distributions in all strips of a Si detector. This assumption was proved to work well if the correction due to the recoil proton tracking in the holding field magnet is applied and if the inelastic $p p$ data is processed in a special way. As result, systematic errors in the beam polarization measured were reduced to $0.5 \%(1.1)$.

Both, the reconstruction of the punch-through protons and the background subtraction were routinely used in the HJET data analysis.

\section{Emulation of the HJET data at EIC}

To anticipate HJET performance at EIC, the $8.9 \mathrm{~ns}$ bunch spacing measurement was emulated using $255 \mathrm{GeV}$ proton beam data acquired in RHIC Run 17. For that, the measured time of each
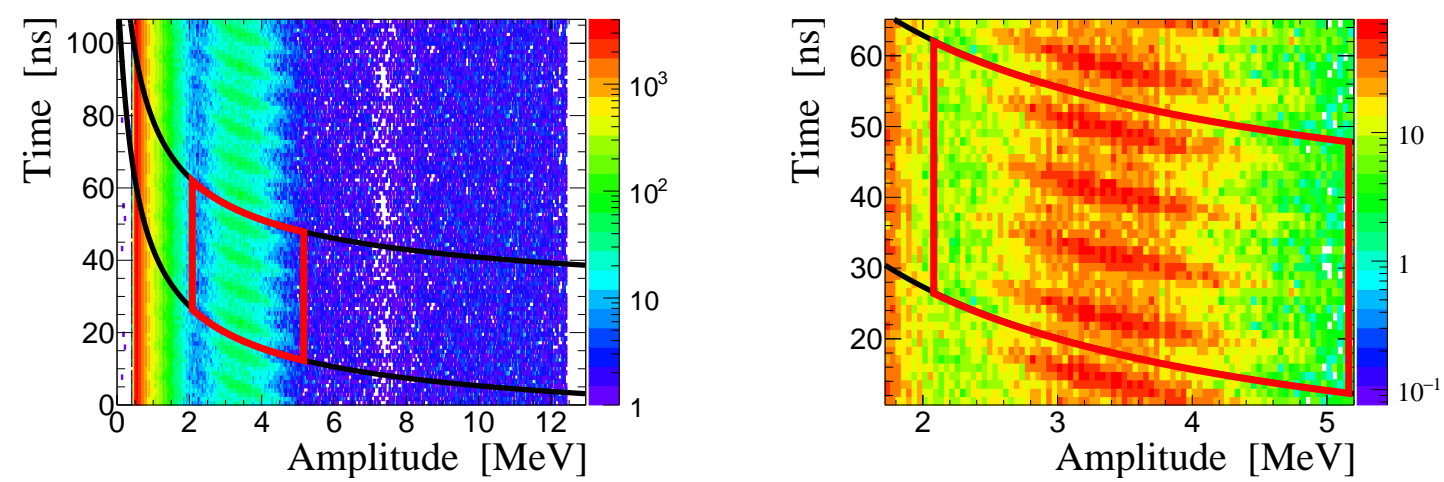

Figure 4: Emulation of the EIC $255 \mathrm{GeV}$ proton beam data with $8.9 \mathrm{~ns}$ bunch spacing. The left histogram time range corresponds to the RHIC bunch spacing of $106.6 \mathrm{~ns}$. The right histogram is the magnified part of the left one. 

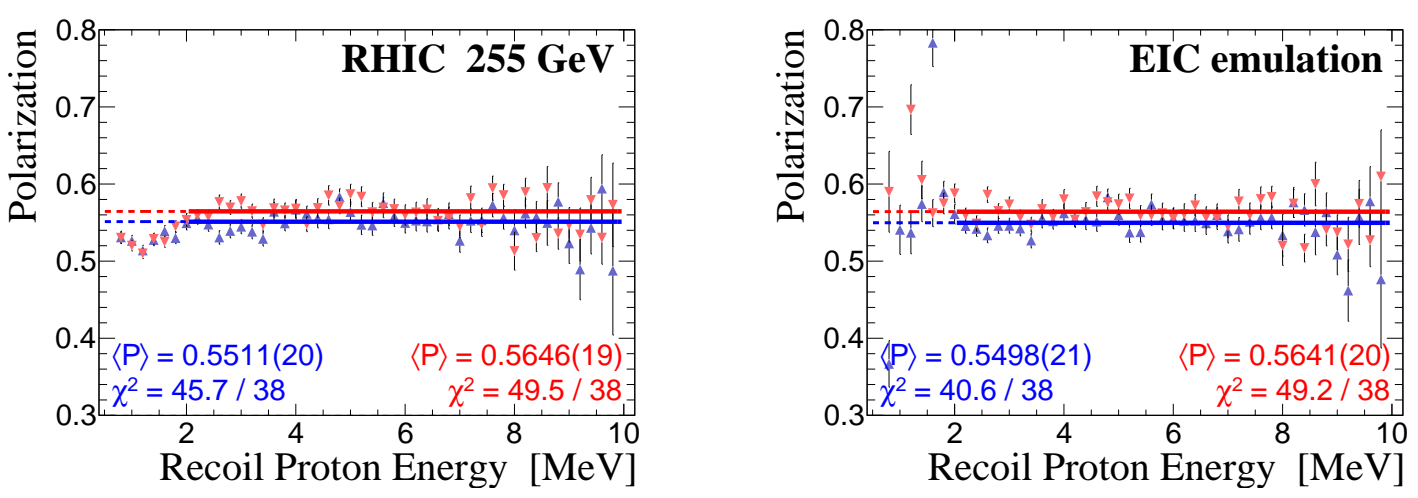

Figure 5: Comparing of the measured beam polarization in RHIC Run 17 and in emulation of the EIC bunch spacing (which was based on the same Run 17 data). Blue and red colors refer to blue and yellow beam measurements, respectively.

event was shifted

$$
t \rightarrow t+k \tau / 12
$$

where $k$ is randomly chosen from $k \in(-1.5,-0,5,0.5,1.5)$ and $\tau=106.6 \mathrm{~ns}$ is the bunch spacing in $p p$ Run 17. This transformation approximates the proposed bunch splitting into four at EIC. In addition, every event was triplicated by the time shifts $\pm \tau / 3$. The resulting time/amplitude distribution is shown in Fig. 4.

Combining four emulated bunches (having the same spin direction at EIC) into one effective bunch, we processed the emulated data using the regular HJET data analysis software. In Fig. 5, the emulated result for the beam polarization determined at EIC is compared with the measurement at RHIC.

Since the same elastic $p p$ events were fit, the statistical errors in both cases are strongly correlated. However, the emulated data is contaminated by background events from other bunches. At RHIC, such background was eliminated by the TOF cuts.

Comparing $P_{\text {beam }}\left(T_{R}\right)$ dependencies in Fig. 5, one finds that, for $T_{R}>2 \mathrm{MeV}$, the 12-fold compression of the bunch spacing at EIC will not alter, within $|\delta P / P| \lesssim 0.3 \%$ uncertainty, the measured beam polarization. This suggests that additional background from other bunches can be well eliminated by the background subtraction method already implemented to the HJET data analysis. Thus, HJET can provide the EIC absolute proton beam polarization measurement with relative accuracy better than $1 \%$.

It should be pointed out, that the situation in the real EIC measurements is expected to be better than the emulation predicts:

- the background is expected to be factor 2 less intensive because there will be only one proton beam crossing HJET;

- the bunch length in EIC will be about order of magnitude shorter than at RHIC, which will allow to use TOF cut more efficiently;

- the emulated bunch spacing ( $8.9 \mathrm{~ns})$ is shorter than that (10.1 ns) proposed for EIC.

Only real Run 17 data with no event preselection (except for the $0.5 \mathrm{MeV}$ threshold trigger) 

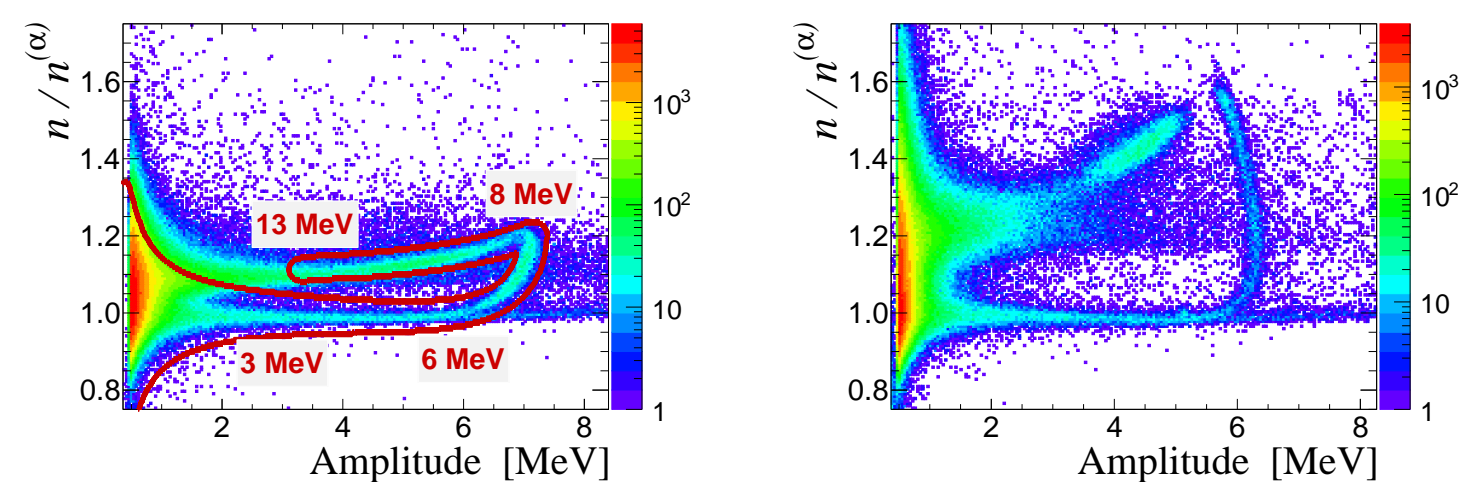

Figure 6: Separation of the stopped and punch-trough recoil protons based on the correlation of the signal waveform parameters $n$ and $A$. All data, including elastic events, prompts, and other background, is displayed. $n^{(\alpha)}$ is value of $n$ obtained in $\alpha$-calibration [6]. Red line specifies the event selection cut used in RHIC Run 17. The correspondence between the $(n, A)$ point on the graph and the recoil proton kinetic energy is labelled for some energies. Right graph shows the same dependence for the altered, $150 \rightarrow 100 \mathrm{~V}$, bias voltage in the Si detector.

was used in the EIC emulation. Therefore, one can expect that the HJET performance at EIC was accurately evaluated. A possible pileup of the signals from different bunches was not considered. However, due to very low event rate of about $\sim 60 \mathrm{~Hz} / \mathrm{strip}$ (mostly prompts), this effect can be neglected. Assuming, that under-threshold event rate is exponentially growing at low energies, we also do not anticipate a significant contribution of such "hidden" events to the pileup. A special consideration is needed for possible signals in the Si detectors electrodynamically induced by the beam bunches. Since no evidence of such signals was observed in RHIC Runs, we also do not expect any visible effect at EIC even though the longitudinal bunch density will be increased by factor 3-4.

\section{Possible improvements of the HJET performance at EIC}

Regardless of the optimistic conclusion about HJET performance at EIC, some improvements are highly recommended.

In the above example, due to data contamination by prompts, the recoil protons with energies below the $T_{R}=2 \mathrm{MeV}$ threshold cannot be used to measure the beam polarization. However, based on our experience in RHIC measurements, these events might be important for calibrations and for monitoring systematic uncertainties.

The signal waveform

$$
W(t) \propto A t^{n} \exp \left(-t / \tau_{s}\right)
$$

fit, helpful to separate stopped and punch-through recoil protons, can also be used to suppress prompts. However, this opportunity was used inefficiently in the above analysis.

The method, illustrated in Fig. 6 (left), is based on comparing the fit values of $n$ and $A$ with the simulated dependencies of these parameters on the recoil proton energy $T_{R}$. In the simulation, which was calibrated using real data [6], for every point $(n, A)$ within the event selection cut, the 


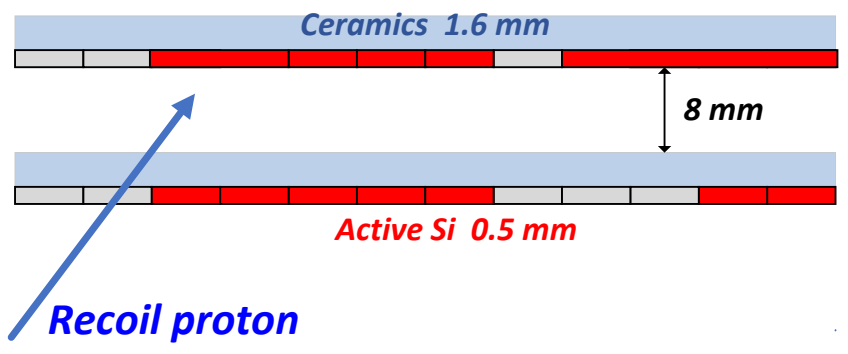

Figure 7: Schematic view of the double layer Si detector prototype which is being tested in RHIC Run 20.

corresponding recoil proton energy was determined. The actual cut used did not suppress prompts if the measured amplitude is $T_{R}<2 \mathrm{MeV}$. This was inessential in RHIC data analysis where prompts are strongly suppressed by TOF. However, more accurate analysis might be critically important at EIC.

Obviously, a tighter $n-A$ cut may reduce the effective $T_{R}$ threshold. The investigation should also include optimization of the bias voltage in silicon detector. Currently, the recoil proton energy and time resolution at HJET is dominated by the electronic noise in the preamplifiers and shapers. Development of new low-noise electronics may also improve the $T_{R}$ threshold.

Using double layer Si detectors to veto prompts at the trigger level is frequently discussed. A prototype of such detector (see Fig. 7), fast-build from the available components was installed in HJET for tests in RHIC Run 20 (low energy Gold beams). The obtained data is being analyzed.

\section{Unpolarized H-jet polarimeter}

The accuracy achieved in the determination of $A_{\mathrm{N}}(t)$ [3] allows one to use a higher density unpolarized hydrogen jet target in a high precision absolute polarimeter, e.g., at EIC.

The unpolarized H-jet thickness of a $10{ }^{14} \mathrm{H}_{2} / \mathrm{cm}^{2}$ (with the jet profile similar to the HJET one) can be produced by the cluster-jet technique, which was developed [7] for the PANDA experiment at GSI. Taking into account higher EIC beam intensity and possibility of recoil proton detectors solid angle increase, 1000 times higher elastic $p p$ event rate can be achieved.

For such a polarimeter, only a $3 \mathrm{~min}$. exposure is needed to determine the beam polarization with statistical accuracy of $\sigma_{P}^{\text {stat }} \sim 1 \%$, while the systematic uncertainty of the measurement is expected to be $\delta^{\text {syst }} P / P \lesssim 1 \%$. Therefore, unpolarized jet can provide accurate measurement of the polarization decay time and, with additional detectors at different azimuthal angles, the beam spin tilt.

However, 1000-fold increase of the data flow will require new data acquisition based on the in-flight fit of the signal waveforms, which, in turn, may affect the quality of the systematic error suppression. At minimum, the jet density should be optimized in this context. The optimization should include comparison of the projected statistical and systematic uncertainties depending on the jet density profile. 
Concurrent operation of the polarized and un-polarized hydrogen jet polarimeters will provide improved statistical accuracy and complimentary control for systematic uncertainties of the measured absolute proton beam polarization.

\section{Summary}

The proton beam precision absolute polarimetry at EIC will include the $200 \mathrm{MeV}$ polarimeter [8] at LINAC and the HJET based polarized and unpolarized hydrogen jet polarimeters at the EIC store ring. The $200 \mathrm{MeV}$ polarimeter does not require any EIC specific changes. An evaluation of HJET performance at EIC and the suggested upgrades were discussed above.

The complete system of EIC polarimeters (e.g. including the polarization profile control, ${ }^{3} \mathrm{He}$ beam polarimeters, etc.) will be discussed elsewhere.

\section{References}

[1] C. Montag et al., in Proc. 10th Int. Particle Accelerator Conf. (IPAC'19), Melbourne, Australia, May 2019, pp. 794-796.

[2] G. Bunce, N. Saito, J. Soffer, and W. Vogelsang, Ann. Rev. Nucl. Part. Sci. 50, 525 (2000).

[3] A. A. Poblaguev et al., Phys. Rev. Lett. 123, 162001 (2019).

[4] V. Ranjbar et al., in Proceedings of 8th International Particle Accelerator Conference (IPAC'17), Copenhagen, Denmark, 2017 (JACoW, Geneva, 2017), pp. 2188-2190.

[5] A. Zelenski et al., Nucl. Instrum. Methods Phys. Res., Sect. A 536, 248 (2005).

[6] A. Poblaguev et al., PoS PSTP 2017, 022 (2018).

[7] A. Taschner, E. Kohler, H. W. Ortjohann and A. Khoukaz, Nucl. Instrum. Meth. A 660, 22 (2011).

[8] A. Poblaguev, G. Atoian, and A. Zelenski, PoS PSTP 2017, 024 (2018). 